\title{
RNA INTERFERNCE (RNAI) FOR SOME GENES FROM BABESIA BOVIS
}

\author{
Mahmoud AbouLaila ${ }^{2^{*}}$, Naoaki Yokoyama ${ }^{1}$, Ikuo Igarashi ${ }^{1}$
}

\author{
${ }^{1}$ Department of Parasitology, Faculty of Veterinary Medicine, University of Sadat City, Sadat \\ City 32511, Minoufiya, Egypt \\ ${ }^{2}$ National Research Center for Protozoan Diseases, Obihiro University of Agriculture and \\ Veterinary Medicine, Inada-Cho, Obihiro, Hokkaido 080-8555, Japan \\ ${ }^{*}$ Corresponding author ; Mahmoud AbouLaila, PhD. \\ Department of Parasitology, Faculty of Veterinary Medicine, University of Sadat City, Sadat City \\ 32511, Minoufiya, Egypt; Phone: 2-048-2603215; E-mail: hethet2004@yahoo.com
}

\begin{abstract}
Babesia parasites are responsible for enormous economic losses to the livestock industry worldwide. There is no vaccine available to protect from the infection. RNA interference (RNAi) has emerged as a simple and incisive technique to study gene functions in a variety of organisms. In this study, we evaluated the effects of RNAi by double-stranded RNA (dsRNA) of the DNA gyrase subunits $A$ and $B$, the DNA-directed RNA polymerase beta subunit (rpo B), and thiostrepton interaction site (ribosomal L11 protein) genes on the in vitro growth of Babesia bovis. The inhibitory effects produced by dsRNAs of DNA gyrase subunit A, DNA gyrase subunit $B$, thiostrepton interaction site (L11) and the DNA-directed RNA polymerase beta subunit (rpo B1and B2) were compared with the effect of drugs target these genes. RNAi directed towards these genes resulted in inhibition of in vitro growth of the parasite. The RT-PCR showed that parasites treated with dsRNAs of DNA gyrase subunit A, DNA gyrase subunit $B$, ribosomal L11, and the DNA-directed RNA polymerase beta subunit (rpo B1 and B2) have blocked expression of corresponding endogenous mRNAs of DNA gyrase subunit A, DNA gyrase B, ribosomal L11, and rpo $B$ genes. These results demonstrate that the RNAi technique disrupted gene function in $B$. bovis and the mechanism of action required further studies to be elucidated.
\end{abstract}

Key words: Apicoplast, RNAi, in vitro, Babesia bovis.

\section{INTRODUCTION}

Babesia parasites are tick-transmitted protozoa. They affect a wide variety of animals and are responsible for enormous economic losses to the livestock industry worldwide (Kuttler, 1988). Moreover, Babesia divergens and Babesia microti have zoonotic importance for human (Krause, 2003). No vaccine was developed for controlling the infection. On the other hand, live Babesia vaccines attenuated by passage through splenectomized cattle resulted in immunity to the vaccinated animals. However, live vaccines have risk, which includes transmission of render pest and vaccination failure (De Vos and Bock, 2000). Several babesicidal drugs that have been in use for years have proven to be ineffective owing to problems related to their toxicity and the development of resistant parasites (Vial and
Gorenflot, 2006). Therefore, there is a need to develop new methods for controlling the infection caused by Babesia parasites. One of the direct ways to determine the biological function of a protein is to knock out the gene by homologous recombination. In recent years, methodologies have been developed that permit transfection of Plasmodium falciparum (Waters et al., 1997) and Babesia bovis (Suarez et al., 2007; Suarez and McElwain, 2008, 2009; Asada et al., 2012; Pedroni et al., 2012) as well as the deletion of specific genes in order to study their function in Plasmodium falciparum (Waters et al., 1997) and in B. bovis (Asada et al., 2012). However, Babesia transfection studies is still in the beginning (Suarez and McElwain, 2010).

RNA interference (RNAi) has emerged as a powerful method for studying gene functions 
in a wide range of organisms. The term RNAi refers to the sequence-specific degradation of mRNA by its homologous double-stranded RNA (dsRNA) (Fire, 1999). Similar phenomena to RNAi have been known for many years in plants, called post-transcriptional gene silencing (PTGS) (Hamilton and Baulcombe, 1999) and in fungi, called 'quelling' (Cogoni et al., 1996). RNAi was first demonstrated in nematodes (Fire, 1999) and was later demonstrated in both unicellular organisms such as Trypanosoma brucei, Plasmodium falciparum, and Toxoplasma gondii (Malhotra et al., 2002; Ngo et al., 1998; Yu et al., 2009) and multicellular organisms such as ticks (Hatta et al., 2007), Brugia malayi, Caenorhabditis elegans, Drosophila, Planaria, Hydra, zebra fish, and mouse (Aboobaker and Blaxter, 2003; Cogoni and Macino, 2000; Maeda et al., 2001). RNAi was applied successfully to study the functions of a large number of genes on a genomic scale in $C$. elegans (Maeda et al., 2001).

Babesia bovis apicoplast is a circular $33 \mathrm{kbp}$ genome and unidirectionally encodes 32 putative protein coding genes, a complete set of tRNA genes, and a small and large subunit rRNA gene as observed with other apicoplast genomes (Brayton et al., 2007). The apicoplast in the apicomplexan parasites, Plasmodium falciparum, Toxoplasma gondii, and Babesia, has typical bacterial housekeeping machinery such as DNA replication, transcription, translation (McConkey et al., 1997). DNA gyrase is a prokaryotic type II topoisomerase involved in untangling DNA during replication. DNA gyrase is nuclear encoded and formed of 2 subunits, DNA gyrase subunit $A$ and DNA gyrase subunit $B$. Ciprofloxacin blocks the prokaryotic DNA replication by inhibiting DNA gyrase and results in linearization of the circular DNA, thus causing death of prokaryotic organisms (Onodera et al., 2001). Rpo B gene is coding for DNAdirected RNA polymerase beta subunit in the apicoplast. Rifampicin blocks bacterial type transcription and abrogates mRNA production by the apicoplast-encoded rpo $\mathrm{B}$ gene (McConkey et al., 1997). The large subunit rRNA (LSU rRNA) is apicoplast encoded and its guanosine triphosphatase (GTPase)-binding domain (ribosomal L11 protein) is responsible for apicoplast translation. The apicoplast translation is inhibited with thiostrepton (McConkey et al., 1997), which also perturbs the proteasome and mitochondria in Babesia (Aboulaila et al., 2012). Pharmaceutical disruption of the genes responsible for the bacterial housekeeping machinery of the apicoplast of Babesia parasites was successfully achieved in vitro by ciprofloxacin, thiostrepton, and rifampicin (AbouLaila et al., 2012). In this study, we present the results of molecular disruption of DNA gyrase Subunit A, DNA gyrase subunit $B$, ribosomal $L 11$, and rpoB genes in Babesia bovis by the RNA interference (RNAi) using double stranded RNA (dsRNA) of target genes.

\section{MATERIALS AND METHODS}

\section{Parasite}

Babesia bovis (B. bovis) Texas strain (Hines et al., 1995) was grown in a micro-aerophilous stationary phase culture system using bovine erythrocytes (RBCs) and GIT medium (Wako Pure Chemicals, Osaka, Japan) (AbouLaila et al., 2010).

DNA extraction, PCR amplification, and Double-stranded RNA in vitro synthesis

B. bovis-infected RBCs with $5 \%$ parasitemia were collected from the culture and washed three times with cold phosphate-buffer saline (PBS) by centrifuging at $1000 \times \mathrm{g}$ for 5 minutes at $4 \stackrel{\circ}{\circ} \mathrm{C}$. DNA was extracted from $200 \mu \mathrm{l}$ infected RBCs using QiAmp DNA Blood Mini kit (QIAGEN, Tokyo, Japan) according to the manufacturer's protocol. DNA concentration was measured using Nano Drop 2000 spectrophotometer (Thermo Fisher Scientific Inc., USA). Primers used in this study were designed based on the sequences of DNA gyrase subunit A, DNA gyrase subunit B, ribosomal protein L11 (Thiostrepton interaction site on the LSU rRNA), rpo B genes, and the sequence of pEGFPn1 plasmid (gene bank accession numbers: XM_001609478, XM_001611055, XM_001610852, XM_001609672, and U55762). For primer design, the amino acid sequences of DNA gyrase subunit $A$ and $B$ 
and the rpo $\mathrm{B}$ were blasted at the protein blast tool at the NCBI to get the active domains of the enzymes (Fig.1 A, a). The gyrase A forward primer 5'GTAATACGACTCACTATAGGTCTGGAGCT GTGCGACGAAC-3' and reverse primer 5'-GTAATACGACTCACTATAGGTATGTGCT CTAATTCGCTGTA- $3^{\prime}$ were used to amplify the nucleotides 300 to 1800 of the DNA gyrase $A$ subunit gene and designed as gyrase $A$ (Fig.1). The gyrase $B$ forward primer 5'-GTAATACGACTCACTATAGG ACTGCGGCTAGTGGATGAAC-3' and reverse-primer-5'-GTAATACGACTCACTATA GGTGACTGCACGTCTCCACCCATG-3' were used to amplify the nucleotides 1200 to 2775 of the DNA gyrase subunit $B$ gene and designed as gyrase $B$ (Fig.1 A). For the rpo $B$ gene, rpo B1 and B2 were selected as target sequences (Fig.1). The rpo B1 forward 5'-GTAATACGACTCACTATAGGATGGAGTT CAATGTTACCG-3' and rpo B1 reverse 5'-GTAATACGACTCACTATAGGTCCACAA GGGGCGCCATCAG-3' primers were used to amplify the nucleotides 1 to 1650 of the rpo $B$ gene. The rpo $B \quad 2 \quad F$ 5'-GTAATACGACTCACTATAGGAGAAGCTA GAATATCTCACC-3' and rpo B2 R 5'-GTAATACGACTCACTATAGGACTGTCTA TAGGAAGACCCAATT-3' primers were used to amplify the nucleotides 2100 to 3849 of the rpo B gene (Fig.1). Ribosomal protein L11 (thiostrepton interaction site) $F$ 5'-GTAATACGACTCACTATAGGATGAAGTT TCTTGGAAGATACC-3' and R 5'-GTAATACGACTCACTATAGGCTACTTTT TCCCGCGTTTCTGC-3' primers were used to amplify the nucleotides 1 to 537 of the ribosomal protein L11 (thiostrepton interaction site) gene. To confirm the specificity of the RNAi mediated by the DNA gyrase $A$ and $B, L 11$, and rpo $B 1$ and $B 2$ dsRNAs, a sequence of GFP at position 672 to 1388 from pEGFPn1 plasmid was amplified by the forward primer 5'-GTAATACGACTCACTATAGGATGGTGA GCAAGGGCGAGGAG-3' and reverse primer-5'-GTAATACGACTCACTATAGGTTA CTTGTACAGCTCGTCCA- $3^{\prime}$. All the primers have the T7 promoter (underlined) at their $5^{\prime}$ end to permit the in vitro transcription of the
RNA strands by the T7 enzyme. PCR was performed in $100 \mu \mathrm{l}$ of a mixture containing $200 \mathrm{ng}$ of the extracted DNA template, 500 $\mathrm{nM}$ of each primer, $200 \mu \mathrm{M}$ of each dNTP, and $5 \mathrm{U}$ of Taq Gold DNA polymerase (Applied Biosystems, Foster City, CA, USA) in a PCR buffer (Applied Biosystems). The reactions were performed at the following temperatures: initial denaturation at $95^{\circ} \mathrm{C}$ for 5 minutes to activate the Taq Gold DNA polymerase followed by 35 cycles ( 1 minute of DNA denaturation, 1 minute of primer annealing (at $65^{\circ} \mathrm{C}$ (gyrase A), $64^{\circ} \mathrm{C}$ (gyrase B), $60.5{ }^{\circ} \mathrm{C}$ (ropB1), $57{ }^{\circ} \mathrm{C}$ (ropB2), $59.4{ }^{\circ} \mathrm{C}$ (L11), and $55{ }^{\circ} \mathrm{C}$ (GFP), and extension at $72^{\circ} \mathrm{C} 1$ (L11 and pEGFPN1), 1.5 (gyrase A) and for 2 minutes (gyrase $B$, rpo $B 1$, and rpo B2), and final extension at $72{ }^{\circ} \mathrm{C}$ for 7 minutes in a Veriti® 96-Well Thermal Cycler (Applied Biosystems, USA). The PCR products were subjected to electrophoresis in $2 \%$ agarose gel and then visualized under an ultraviolet (UV) light and stained with eithedium bromide (Sigma-Aldrich). The PCR products were purified using QIAquick PCR purification kit (QIAGEN, Tokyo, Japan). The concentration of the purified products was quantified using Nano Drop 2000 spectrophotometer (Thermo Fisher Scientific Inc., USA). The dsRNA was synthesised from about $1 \mu \mathrm{g}$ of each of the purified PCR products of gyrase A, gyrase B, rpo B1, rpo B2, L11, and GFP using T7 RiboMAX Express RNAi system (Promega, Madison, USA) according to the manufacturer's instructions. The dsRNA was resuspended in $0.1 \%$ diethylpyrocarbonate (DEPC) treated water. The purity of synthesized dsRNA was checked by $1 \%$ agarose gel electrophoresis and the concentration was quantified by spectrophotometer (Thermo Fisher Scientific Inc., USA). The dsRNA aliquots were stored in $-80 \cong \mathrm{C}$ until used.

\section{RNAi in vitro growth inhibition assay}

The in vitro growth inhibition assay for RNAi followed a method previously described (AbouLaila et al., 2010; Malhotra et al., 2002) with some modifications. Parasite-infected RBCs were diluted with uninfected RBC to obtain $1 \%$ parasitemia. Twenty $\mu$ l of RBC with $1 \%$ parasitemia was dispensed to a 96 -well 
microtiter plate together with $200 \mu$ l of the culture medium containing the indicated concentration of double stranded RNA (dsRNA) $(10$ and $50 \mu \mathrm{g} / \mathrm{ml}$ for gyrase $A$, gyrase $B, L 11$, rpo $B 1$, and rpo $B 2$ ). A mixture of $10 \mu \mathrm{g} / \mathrm{ml}$ of each of gyrase $A$ and gyrase $B$ and another mixture of $10 \mu \mathrm{g} / \mathrm{ml}$ of each rpo B1 and rpo B2 were used. Then, plates were incubated at $37^{\circ} \mathrm{C}$ in a humified multi-gas water-jacketed incubator. For the negative control, cultures without the dsRNA containing only DEPC treated water and another containing a control dsRNA for GFP (Gong et al., 2008) in a similar concentration like the highest concentration were prepared. For the positive control, cultures containing ciprofloxacin, thiostrepton, and rifampicin at a concentration of $50 \mu \mathrm{g} / \mathrm{ml}$ were used. The experiments were carried out in triplicate for 3 separate trials for a period of four days. The culture medium was replaced daily with 200 $\mu \mathrm{l}$ of a fresh medium containing the appropriate concentration of the dsRNA. Parasitemia was monitored daily by counting the parasitized RBCs to 1,000 in Giemsa-stained thin blood smears. Morphological changes in the parasites from treated cultures were reported by light microscope.

\section{Confirmation of gene silencing by reverse transcription PCR (RT-PCR)}

$B$. bovis was cultured in 24 well culture plates in bovine RBCs and GIT medium. The culture was treated with $100 \mu \mathrm{g} / \mathrm{ml}$ of dsRNA of gyrase $A$, gyrase $B$, ribosomal protein $L 11$, rpo B1 and B2 and a control GFP dsRNA in triplicate for 2 days. RBCs were collected and washed with PBS. The total RNA was extracted using TRI® reagent (Sigma-Aldrich, USA) according to the supplied protocol. The RNA concentration was measured using spectrophotometer (Thermo Fisher Scientific Inc., USA). The RT PCR was conducted using the PrimeScript ${ }^{\mathrm{TM}}$ One-Step RT-PCR kit (Takara, Japan) according to the manufacturer's protocol. $150 \mathrm{ng}$ of total RNA from the treated cultures and from the control were used in the amplification of the target sequences with the specific primers for the targeted sequences that were used in the PCR reactions above (this study) and for the full length DNA gyrase subunit $A$, DNA gyrase subunit $B, L 11$ protein, and rpo $B$ genes (AbouLaila et al., 2012). Gyrase A forward primer 5'-CTGGTTTATTATATATGAACCC-3 and reverse primer 5'-CATTTTAGCAAAT ATTAGTTA-3' were used to amplify the DNA gyrase subunit A. Gyrase B forward primer 5'-CCCGCCTCACTGCTACTGGCAGATG-3' and reverse primer 5'-CTACCTATCTAGGTCATGTAGC-3' were used to amplify the DNA gyrase subunit $B$. L11 forward primer 5'-ATGAAGTTTCTTGGAAGATACC-3' and reverse

primer-5'-CTACTTTTTCCCGCGTTTCTGC$3^{\prime}$ ' were used to amplify the L11 protein gene. Rpo B forward primer 5'-ATGGAGTTCAATGTTAACCGT-3' and reverse-primer-5'-TCAACTGTCTATAGGAA GACCC-3' were used to amplify the rpo B gene. $B$. bovis tubulin beta chain gene specific primers, forward-5'-ATGAGAGAAATCGTACACATCC $-3^{\prime}$ and reverse 5'-TCAATAATCATTTACCA TGTCATCGG-3' (AbouLaila et al., 2012), B. bovis small subunit rRNA (ssrRNA) gene primers foreword 5'-TAGTCATATGCTTGTCTTAA-3' and reverse 5'-GGAAACCTTGTTACGATTC-3' (AbouLaila et al., 2012), and B. bovis Rhoptry-Associated Protein 1 (rap-1) foreword-5'-CACGAGCAAGGAACTACCGA TGTTGA-3' and reverse 5'-CCAAGGACCTTCAACGTACGAGGTCA3' primers (Figueroa et al., 1993) were used as controls for the transcription of the targeted genes from the dsRNA treated and GFP control cultures. The reverse transcription reaction was carried out at $50^{\circ} \mathrm{C}$ for 30 minutes, and then PCR was repeated for 30 cycles under the following conditions: 30 seconds on denaturation at $94{ }^{\circ} \mathrm{C}, 30$ seconds of primer annealing (at $50{ }^{\circ} \mathrm{C}$ gyrase A, $62{ }^{\circ} \mathrm{C}$ gyrase B, $59.4{ }^{\circ} \mathrm{C}$ L $11,56{ }^{\circ} \mathrm{C}$ rpo B, $55^{\circ} \mathrm{C}$ RAP $-1,57^{\circ} \mathrm{C}$ ssrRNA and $54^{\circ} \mathrm{C}$ tubulin beta chain), and elongation at $72{ }^{\circ} \mathrm{C}$ for 0.45 (L11 and RAP-1), 2 (tubulin beta chain and ssrRNA), 3 (gyrase A and B), , and 4 (rpo B) minutes. The PCR products were subjected to electrophoresis in $2 \%$ agarose gel and stained with eithedium bromide. 


\section{Statistical analysis}

JMP statistical software version 5.1(SAS Institute Inc., USA) was used to compare the means in the in vitro inhibition assay by one

\section{RESULTS AND DISCUSSION PCR and dsRNA production}

rpo B1, rpo B2 and GFP were successfully amplified with PCR (data not shown).. The in vitro transcription using T7 enzyme resulted in target specific dsRNA of 1501, 1576, 1650, 1750,538 , and $716 \mathrm{bp}$ for each of gyrase $A$, gyrase B, rpo B1, rpo B2, L11, and GFP genes, respectively (Fig.1).

\section{In vitro growth inhibition assay}

$B$. bovis growth (Fig. 2) from an initial parasitemia of $1 \%$ was significantly (ANOVA) inhibited at $10 \mu \mathrm{g} / \mathrm{ml}$ and $50 \mu \mathrm{g} / \mathrm{ml}$ concentrations of gyrase $A$, gyrase $B$, rpo B1 and B2, and L11 (Fig. 2). Treatment with a mixture formed of $10 \mu \mathrm{g} / \mathrm{ml}$ gyrase $A$ and 10 $\mu \mathrm{g} / \mathrm{ml}$ gyrase B significantly inhibited the growth (ANOVA, $P<0.01$ ). The mixture formed of $10 \mu \mathrm{g} / \mathrm{ml}$ rpo $B 1$ and $10 \mu \mathrm{g} / \mathrm{ml}$ rpo B2 resulted in significant inhibition of the growth (ANOVA, $P<0.01$ ) (Fig. 2). The single treatment with $10 \mu \mathrm{g} / \mathrm{ml}$ or $50 \mu \mathrm{g} / \mathrm{ml}$ of each of the dsRNA of gyrase A, gyrase B, rpo B1, rpo B2, and L11 (Tukey-Kramer HSD) did not show significant difference. There was no RT- PCR

B. bovis cultures treated with $100 \mu \mathrm{g} / \mathrm{ml}$ dsRNA of gyrase A, gyrase B, L11, rpo B1 and $\mathrm{B} 2$, and GFP for 2 days; then, reverse transcription PCR for total RNA extracted from these cultures showed that the way analysis of variance (ANOVA) followed by all pairs Tuckey-Kramer HSD and considered to be significantly different when $\mathrm{P}<0.01$.

Target sequences of 1501, 1576, 1650, 1750, 538 , and 716 bp specific for gyrase A, gyrase significant difference between the single treatment with $10 \mu \mathrm{g} / \mathrm{ml}$ or $50 \mu \mathrm{g} / \mathrm{ml}$ of each dsRNA and the mixture formed of $10 \mu \mathrm{g} / \mathrm{ml}$ gyrase $A$ and $10 \mu \mathrm{g} / \mathrm{ml}$ gyrase $B$ dsRNAs and the mixture formed of $10 \mu \mathrm{g} / \mathrm{ml} \mathrm{rpo} \mathrm{B1}$ and 10 $\mu \mathrm{g} / \mathrm{ml}$ rpo B2 dsRNAs (Tukey-Kramer HSD) (Fig. 2). Ciprofloxacin, thiostrepton, and rifampicin at a $50 \mu \mathrm{g} / \mathrm{ml}$ concentration significantly suppressed the growth on the first day of culture than the control and dsRNA treatments. Light microscope observation showed that the treatment with dsRNA of gyrase A (Fig. 3B), gyrase B (Fig. 3 C), gyrase $A$ and $B$ mixture (Fig. 3D), L11 (Fig. $3 \mathrm{E}$ ), rpo B1 (Fig.3 F), rpo B2 (Fig. $3 \mathrm{G}$ ), and rpo $\mathrm{B} 1$ and rpo $\mathrm{B} 2$ mixture (Fig. $3 \mathrm{H}$ ) resulted in morphological changes in the treated parasites than the GFP dsRNA control cultures. Parasites in treated cultures appeared degenerated without cytoplasm, swollen, and there was a high numbers of abnormally multidividing forms (Fig. 3).

transcription of gene of DNA gyrase subunit $A$ (Fig. 4 A, lane 2), DNA gyrase subunit $B$ (Fig. $4 \mathrm{~A}$, lane 6), rpo B (Fig. $4 \mathrm{~A}$, lane 14), and ribosomal L11 protein (Fig. 4 B, lane 2 ) genes was inhibited than in the GFP dsRNA control (Fig.5A, lanes 1, 5, B, lanes, 1, 5, and

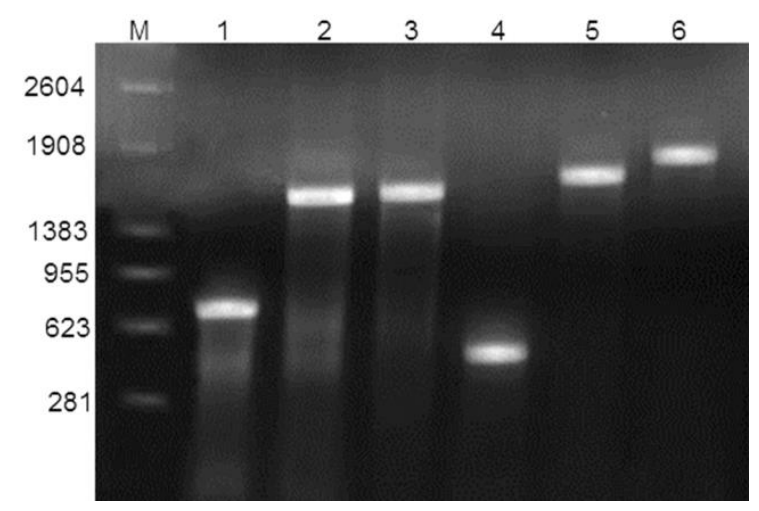

Fig.1. Denaturing agarose gel electrophoresis of double stranded RNAs of DNA gyrase subunit A, DNA gyrase subunit 
B, ribosomal L11 protein, DNA directed RNA polymerase subunit B (rpo B) part 1 (rpo B1) and part 2 (rpo B2), and GFP. Lane 1, GFP dsRNA; lane 2, gyrase A dsRNA; lane 3, gyrase B dsRNA; lane 4, L11 protein dsRNA; lane 5, rpo B1 dsRNA; lane 6, rpo B2 dsRNA. $M$ is molecular weight marker.

(A)

(B)
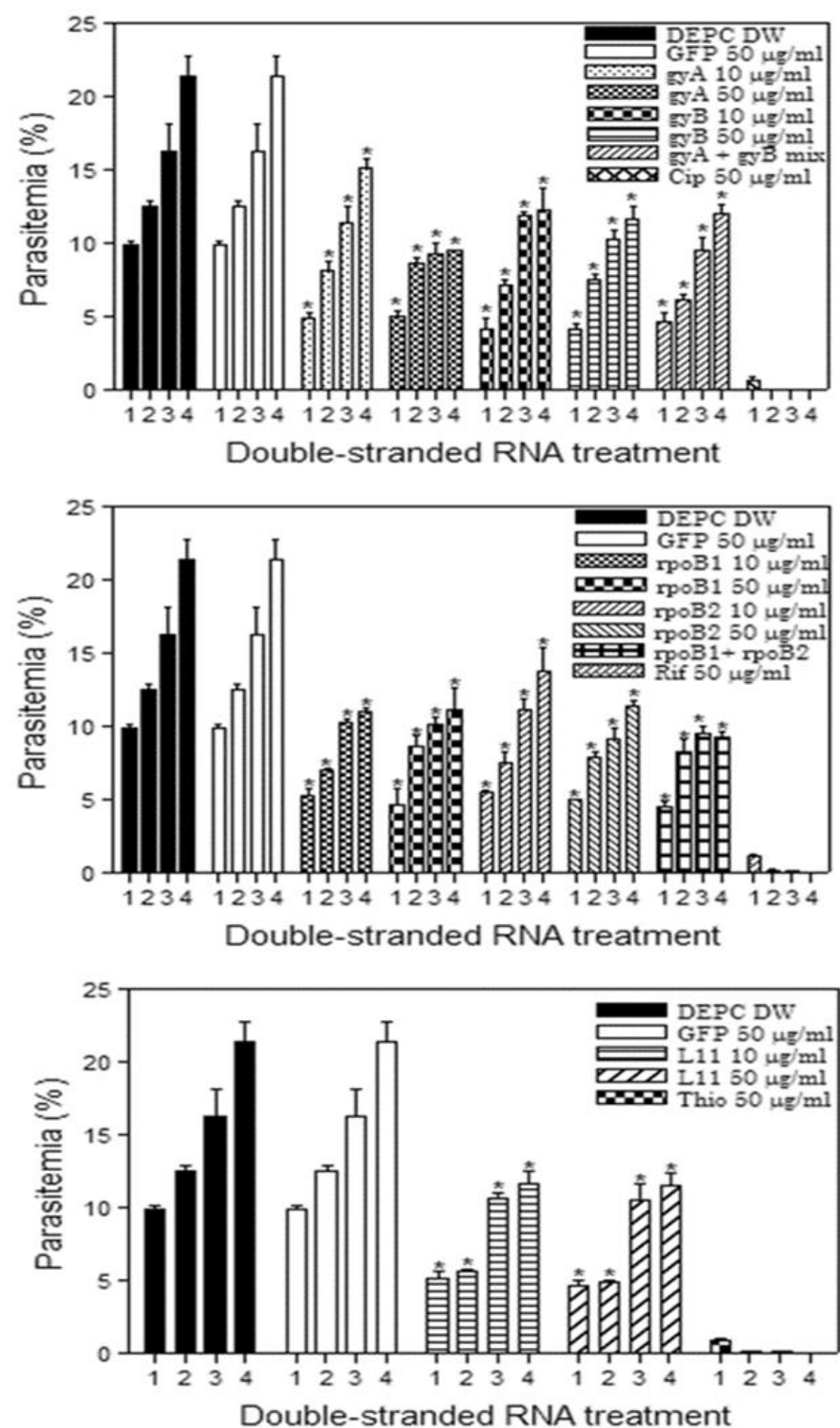

Fig.2. Inhibitory effect of RNAi on the in vitro growth of Babesia bovis. Each value represents the mean \pm standard deviation of three separate experiments carried out for 4 days in triplicate. Asterisks, indicate significant difference $(P<$ 0.01 ) between dsRNA-treated and the dsRNA for GFP and DEPC DW control cultures of $B$. bovis.

C, lane 1 ). Dissimilarly, the transcription of B. bovis tubulin beta chain gene was not affected by the dsRNA treatment (Fig. $4 \mathrm{~A}$, lanes 4,8 , B, lane 4,8 , and $C$, lane 4) than in the GFP dsRNA control culture (Fig. 4A, lanes 3, 7, B, lane 3, 7, and C, lane 3). Rap-1 transcripts were not affected by the dsRNA treatment (Fig.4A, lanes 11, 13, B lanes 9, 15, and $C$, lane 5) than in GFPdeRNA control (Fig.4A, lanes 12, 14, B lanes 10, 16, and C, lane 6).

Transcripts of ssrRNA gene were not affected by the dsRNA treatment (Fig.5A, lanes 9, 15, $B$ lanes, 11, 13 and $C$, lane 7) than GFPdeRNA control culture (Fig.4A, lanes 10, 16, B lanes, 12, 14 and C, lane 8). RT-PCR 
using specific primers for the target sequences showed no transcription of the targeted sequences of gyrase $A$, gyrase B, rpo B1, rpoB2, and ribosomal L11 protein. In contrast, transcripts of tubulin beta chain, rap-1, and ssrRNA gene were not affected by
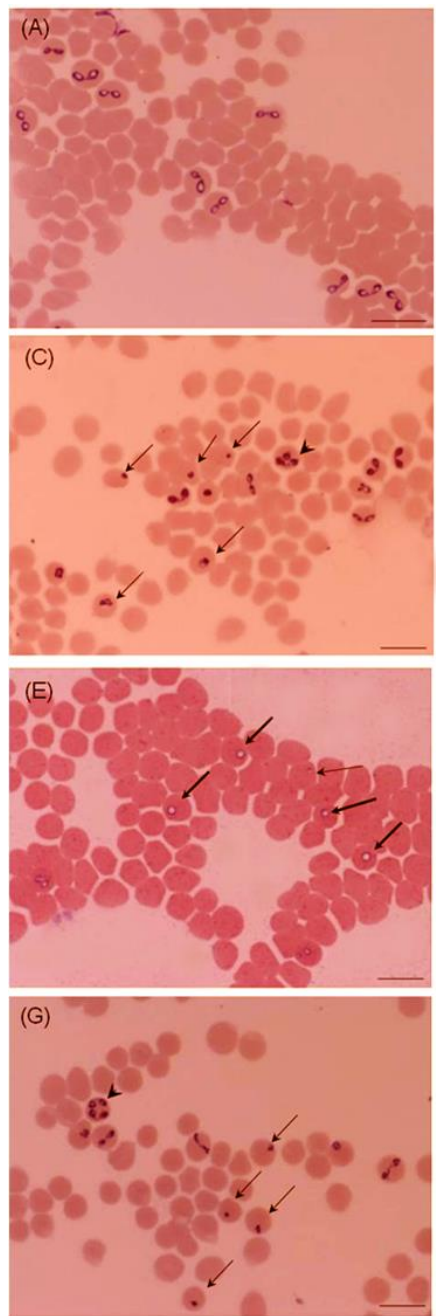

Fig.3. Double stranded RNA-treated Babesia bovis in an in vitro culture: (A) GFP dsRNA control. (B) Treated with dsRNA of gyrase A. (C) Treated with dsRNA of gyrase B. (D) Treated with dsRNA of gyrase A and gyrase B. (E) Treated with dsRNA of ribosomal L11 protein. (F) Treated with dsRNA of rpoB1. (G) Treated with dsRNA of rpoB2. (H) Treated with dsRNA of rpoB1 and rpoB2. RNAi-treated cultures showed a higher number of degenerated parasites (thin arrows), swollen parasites (thick arrows), and abnormally multidividing forms (arrowheads). Bars, $10 \mu \mathrm{m}$.
These genes were targeted by ciprofloxacin, rifampicin, and thiostrepton leading to inhibition of $B$. bovis growth (AbouLaila et al., 2012). Inhibition of $B$. bovis growth by in vitro treatment with the dsRNA of gyrase $A$, gyrase $B$, L11 protein, rpo B1 and B2 genes was revealed. All the concentrations of dsRNA resulted in significant inhibition of $B$. bovis growth. Alternatively, no significant difference was observed among the treated culture with $10 \mu \mathrm{g} / \mathrm{ml}$ and $50 \mu \mathrm{g} / \mathrm{ml}$. treatment with dsRNA targeting the 2 targeted sequences of rpo $\mathrm{B}$,
dsRNA treatments.

In the present study, we attempted RNAi in Babesia bovis, an intracellular parasite using dsRNAs for DNA gyrase, DNA-directed RNA polymerase beta subunit, and thiostrepton interaction site (ribosomal L11 protein).
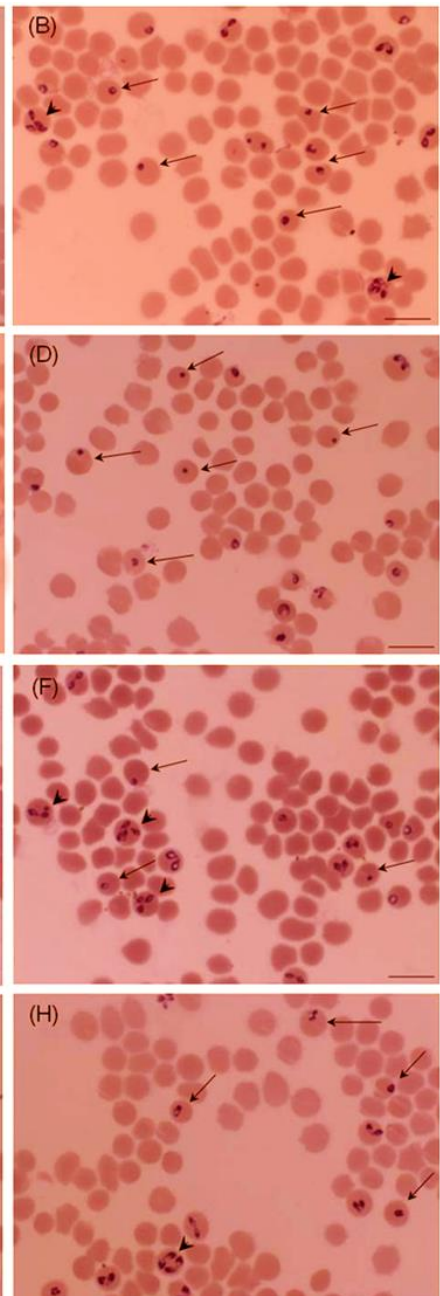

rpo B1 and B2, either alone at $10 \mu \mathrm{g} / \mathrm{ml}$ or in a mixture formed of $10 \mu \mathrm{g} / \mathrm{ml}$ of each of them could not produce significant differences. Similarly, treatment with dsRNA at $10 \mu \mathrm{g} / \mathrm{ml}$ for gyrase A or gyrase B was similar to the mixture formed of $10 \mu \mathrm{g} / \mathrm{ml}$ of each the dsRNA to silence the DNA gyrase. This might be owing to silencing a part of gene by RNAi leads to the destruction of the whole gene transcript and this in a good agreement with Hatta et al., (2007), who silenced the whole gene encoding cytosolic leucine 
aminopeptidase of the Hemaphysalis longicornis by dsRNA of a partial-length $\mathrm{N}$-terminal domain of the gene. Treatment with dsRNA of GFP from pEGFPn1 and DEPC treated water had no effect on $B$. bovis growth indicating that the inhibitory effect was due to treatment with dsRNA of gyrase A, gyrase $B, L 11$, and rpo B1and B 2. The dsRNA treatment resulted in sever morphological changes in the parasites from treated cultures, but not in the parasites from DEPC DW or a non-specific GFP dsRNA treated cultures, indicating specific inhibition of $B$. bovis by RNAi.
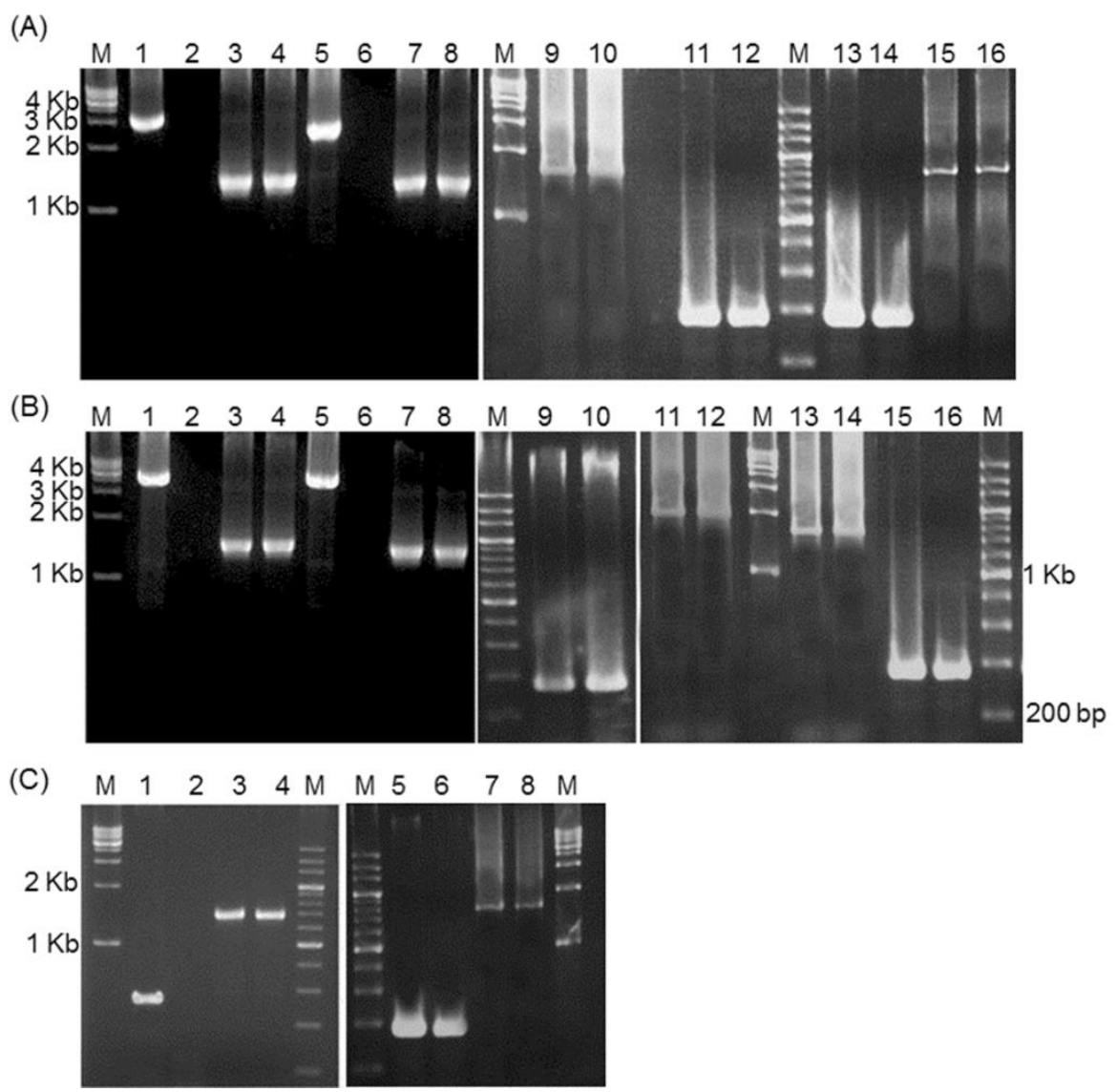

Fig.4. Reverse transcription-PCR (RT-PCR) for the Babesia bovis cultures treated with $100 \mu \mathrm{g} / \mathrm{ml}$ dsRNA of DNA gyrase subunit $A$ and $B$, thiostrepton interaction site (ribosomal protein L11), rpo B1 and B2, and GFP. Tubulin beta chain, rap-1, and ssrRNA genes were used as controls for dsRNA and dsRNA of GFP treatments. (A) DsRNA treatment of gyrase A (lane 2), gyrase B (lane 6). (B) DsRNA treatment of rpo B1 (lane 2), rpo B2 (lane 6), and control (lanes 1, 5, 9, and 13). (C) DsRNA treatment of thiostrepton interaction site (ribosomal L11 protein) (lane 2) and GFP dsRNA control (lane 1). Tubulin beta chain (A and $B$, lanes 3,4,7,8 and $C$, lanes 3, 4), rap-1 (A, lanes 11,12, 13, 14, B, lanes 9, 10, 15, 16, and C, lanes 5, 6), and ssrRNA (A, lanes 9, 10, 15, 16, B, lanes11,12, 13, 14, and C, lanes 7,8 ) genes from dsRNA-treated and dsRNA of GFP-treated cultures. $M$ is molecular weight marker.

We also compared the effects produced by DNA gyrase, L11, and rpo B dsRNAs on $B$. bovis parasites with the effects of ciprofloxacin, thiostrepton, and rifampicin, well-known inhibitors of DNA gyrase, apicoplast translation, and rpo $B$, respectively. Our study showed that dsRNA treatment reduced the growth but the inhibition was lower than the inhibition resulted from drug treatments. This might be due to dsRNA was not able to enter through cell wall and only the small molecules were able to penetrate into the cells, resulted from its break down in the medium. The results are covenant with previous study on plasmodium falciparum (Malhotra et al., 2002) as RNAi using dsRNA not completely eliminate the parasites that might be as a result of technical limits of the 
technique.

The gene silencing by the RNAi was confirmed by RT-PCR. The inhibitory effects produced by dsRNA treatment of parasites coincided with a marked reduction in the levels of endogenous mRNA homologous to the dsRNA in these parasites, which is a characteristic feature of RNAi. The RT reactions from parasite cultures treated with dsRNA of gyrase A, gyrase B, L11, rpo B1, and rpo B2 showed that no amplifications were reported for either the target sequences or full length genes of DNA gyrase subunit $A$, DNA gyrase subunit $B, L 11$, rpo $B$ indicating the disruption of the mRNA transcripts of these genes by the RNAi. In contrast, the control tubulin beta chain, rap-1, and ssrRNA genes were successfully amplified from the dsRNA treated cultures indicated that the mRNA transcripts for tubulin beta chain, rap-1, and ssrRNA were not affected; therefore, the dsRNA treatment is specific for the targeted genes. The DNA gyrase subunit A, DNA gyrase subunit $B, \quad L 11$, rpo $B$ were successfully amplified by the RT PCR from GFP dsRNA control cultures as well as the tubulin beta chain, rap-1, and ssrRNA controls; therefore, the RNAi is specific effective tool in the disruption of gene functions in B. bovis.

In this study, we used in vitro dsRNA disruption of $B$. bovis and this method depends on the uptake of the dsRNA by the parasites and our results were in covenant with the in vitro dsRNA disruption of $P$. falciparum (Malhotra et al., 2002). Furthermore, the results of the present study is consistent with previous RNAi studies including soaking of whole $C$. elegans (Tabara et al., 1998) and Brugia malayi (Aboobaker and Blaxter, 2003) in a solution containing dsRNA or the in vitro incubation of Drosophila S2 cells with dsRNA (Clemens et al., 2000), all showing the ability of dsRNA to cross cell boundaries and pass through several types of tissues.

The use of RNAi in $B$. bovis has several advantages over other methods requiring the introduction of DNA into cells. Transfection experiments require more knowledge of the full gene sequence and its flanking regions while dsRNA corresponding to any gene fragments is sufficient to produce the interference effect in the RNAi (Ullu and Tschudi, 2000). Furthermore, the use of RNAi is technically simple and quick achieving results within 2-3 days. On the other hand, selective gene 'knock-outs' by transfection technology requires longer time.

RNAi in $B$. bovis also provides a tool for studying the functions of a number of genes together, as shown in the present study. With the availability of a large amount of sequence information for $B$. bovis, RNAi-based functional genomics might be a useful technique for investigating the biological functions of novel genes. Finally, given the gene-specific feature of RNAi and the ease of inducing RNAi, this methodology may also play a role in the development of therapeutic applications against Babesia infections.

The discovery of RNAi is one of the marvelous advances in molecular genetics in the last decade (Fjose et al., 2001; Yu et al., 2009). RNA interference (RNAi) is becoming an increasingly powerful post-transcriptional gene silencing (PTGS) technique that is providing insight into gene function (Elbashir et al., 2001; Hannon, 2002) and consequently the reliability of gene identity rose by homology search of the databases. The RNAi process involves an ATP-dependent production of small, $\approx 21-25$ nt, interfering RNA molecules (siRNAs) from double stranded RNA (dsRNA) and is believed to be responsible for targeting and destroying specific mRNAs facilitated by the formation of the RNA-induced silencing complex (RISC) (Moissiard et al., 2006). The RISC RNA molecules complementary to the mRNAs seem to work as a guide recruiting a ribonuclease that consequently cleaves only specific mRNAs (Aigner, 2008; Grishok et al ., 2001). RNAi is a two-step mechanism and both mechanisms and the components of dsRNA-induced gene silencing in a diverse group of organisms are conserved. Two sets of genes coding for proteins implicated in RNAi have been identified in different organisms. The first set of proteins is the one with homology to tomato RNA-directed RNA polymerase (RdRP): Neurospora QDE-1, C. 
elegans EGO-1 and Araabidopsis SGS-2/SDE-1. The second set of proteins involved in RNAi includes Arabidopsis AGO-1, Neurospora QDE-2 and C. elegans RDE-1 (Malhotra et al., 2002; Fagard et al., 2000; Elbashir et al., 2001; Grishok et I., 2001; Lee et I., 2010). Some related sequences were reported in $P$. falciparum through the blast search tool at the PlasmoDB.org (Malhotra et al., 2002). Instead, some recent studies mentioned that RNAi pathway important components for instance Dicer and Argonaute genes are absent from $P$. falciparum (Atayde et al., 2011; Baum et al., 2009; Kolev et al., 2011; Xue et al., 2008). Furthermore, no related sequences were detected by blast search of the sequences of these genes with the $B$. bovis server at www.sanger.ac.uk/cgi-bin/blast/submitblast/b bovis and this is compatible with Batista and Marques, (2011); therefore, further studies are required for the understanding of the mechanism of RNAi by dsRNA in Babesia parasites

In conclusion, the present study shows that the RNAi technique disrupted the transcripts of targeted genes and affected the growth of parasites; however, the machinery in $B$. bovis required further studies to be elucidated.

\section{ACKNOWLEDGEMENT}

This study was supported by Ministry of Higher Education of Egypt, Ministry of Education, Culture, Research, Sports, Science, and Technology of Japan, and the Japan Society for the Promotion of Science (JSPS).

\section{REFERENCES}

Aboobaker, A. A. and Blaxter, M. L. (2003). Use of RNA interference to investigate gene function in the human filarial nematode parasite Brugia malayi. Mol. Biochem. Parasitol. 129: 41-51.

AbouLaila, M., Tserendorj, M., Sivakumar, T., Ueno, A., Nakano, Y., Yokoyama, M., Yoshinari, T., Nagano, D., Katayama, K., EL-Bahy, N., Yokoyama, N., and Igarashi, I. (2012). Apicoplast-targeting antibacterials inhibit the growth of Babesia parasites. Antimicrob.Agents
Chemother. 56: 3196-3206.

AbouLaila, M., Nakamura, K., Yadav, G., Yokoyama, M., and Igarashi, I. (2010). Evaluation of the in vitro growth-inhibitory effect of epoxomicin on Babesia parasites. Vet. Parasitol.167 (1): 19-27.

Aigner, A. (2008). Cellular delivery in vivo of siRNA-based therapeutics. Curr. Pharm. Des. 14: 3603-3619.

Asadaa, M., Tanakaa, M., Goto, Y., Yokoyama, N., Inoue, N., and Kawazu, S.-I. (2012). Stable expression of green fluorescent protein and targeted disruption of thioredoxin peroxidase-1 gene in Babesia bovis with the WR99210/dhfr selection system. Mol. Biochem. Parasitol. 181: 162-170.

Atayde, V. D., Tschudi, C., and Ullu, E. (2011). The emerging world of small silencing RNAs in protozoan parasites. Trends Parasitol. 27: 321-326.

Batista, T. M., and Marques, J. M. (2011). RNAi pathways in parasitic protists and worms. J. Proteomics 7 4:1504-151 4.

Baum, J., Papenfuss, A T., Mair, G. R., Janse, C. J., Vlachou, D., Waters, A. P., Cowman, A. F., Crabb, B. S. and de Koning-Ward, T. F. (2009). Molecular genetics and comparative genomics reveal RNAi is not functional in malaria parasites. Nucl. Acids Res. 37: 3788-3798.

Brayton, K., Lau, A., Herndon, D., Hannick, L., Kappmeyer, L., Berens, S., et al., (2007). Genome sequence of Babesia bovis and comparative analysis of apicomplexan hemoprotozoa. PLoS Pathog. 3: 1401-1413.

Clemens, C.J., Worby, C.A., Simonson-Leff, N., Muda, M., Maehama, T., Hemmings. B.A., and Dixon, J.E. (2000). Use of double-stranded RNA interference in Drosophila cell lines to dissect signal transduction pathways. P.N.A. S. 97: 6499-6503.

Cogoni, C. and Macino, G. (2000). Post-transcriptional gene silencing across kingdoms. Curr. Opin. Genes Dev. 10: 638-643. 
Cogoni, C., Irelan, J.T., Schumacher, M., Schmidhauser, T.J., Selker, E.U., and Macino, G. (1996). Transgene silencing of the ala-1 gene in vegetative cells of Neurospora is mediated by a cytoplasmic effector and does not depend on DNA-DNA interactions or DNA methylation. EMBOJ. 15: 3153-3163.

De Vos, A. and Bock, R.E. (2000). Vaccination against bovine babesiosis. Ann. N. Y. Acad. Sci. 916: 540-545.

Elbashir, P.J., Lendeckel, W., and Tuschl, T. (2001). RNA interference is mediated by 21- and 22-nucleotide RNAs. Genes Dev. 15: 188-200.

Kolev, N. G., Tschudi, C., and Ullu, E. (2011). RNA interference in protozoan parasites: achievements and challenges. Eukaryotic Cell 10: 1156-1163.

Fagard, M., Boutet, S., Morcel, J., Bellini, C., and Vaucheret, H. (2000). AGO1, QDE-2 and RDE-1 are related proteins required for post-transcriptional gene silencing in plants, quelling in fungi, and RNA interference in animals. P.N.A.S. 97: 11650-11654.

Figueroa, J.V., Chieves, L.P., Johnson, G.S., and Buening, G.M. (1993). Multiplex polymerase chain reaction based assay for the detection of Babesia bigemina, Babesia bovis and anaplasma marginale DNA in bovine blood. Vet. Parasitol. 50: 69-81.

Fire, A., 1999. RNA-triggered gene silencing. Trends Gen. 15: 358-363.

Fjose, A., Ellingsen, S., Wargelius, A., and Seo, H.-C. (2001). RNA interference: Mechanisms and applications. Biotechnol. Ann. Rev. 7: 31-57.

Gong, H., Liao, M., Zhou, J., Hatta, T., Huang, P., Zhang, G., Kanuka, H., Nishikawa, Y., Xuan, X., and Fujisaki, K., (2008). Gene silencing of ribosomal protein $\mathrm{PO}$ is lethal to the tick Haemaphysalis longicornis. Vet.Parasitol.151 (2-4): 268-278.
Grishok, A., Pasquinelli, A.E., Conte, D., Li, N., Parrish, S., and Ha, I., et al., (2001). Genes and mechanisms related to RNA. Interference regulates expression of the small temporal RNAs that control $C$. elegans development timing. Cell 106: 23-34.

Hamilton, A.J. and Baulcombe, D.C. (1999). A species of small RNA in post transcriptional gene silencing in plants. Science 286: 950-952.

Hannon, G.J. (2002). RNA interference. Nature 418: 244-251.

Hatta, T., Umemiya, R., Liao, M., Gong, H., Harnnoi, T., Tanaka, M., Miyoshi, T., Boldbaatar, D., Battsetseg, B., Zhou, J., Xuan, X., Tsuji, N., Taylor, D., and Fujisaki, K. (2007). RNA interference of cytosolic leucine aminopeptidase reduces fecundity in the hard tick, Haemaphysalis longicornis. Parasitol. Res. 100: 847-854.

Hines, S., Palmer, G., Brown, W., McElwain, T., Suarez, C., Vidotto, O., and Rice-Ficht, A. (1995). Genetic and antigenic characterization of Babesia bovis merozoite spherical body protein Bb-1. Mol. Biochem.Parasitol.69: 149-159.

Krause, P. J. (2003). Babesiosis Diagnosis and Treatment. Vector-borne Zoonotic Dis. 3: $45-51$.

Kuttler, K. (1988). World-wide impact of babesiosis. In: Ristic, M. (ed.). Babesiosis of domestic animals and man. CRC Press Inc., Florida, pp. 1-22.

Lee, H.C., Aalto, A.P., Yang, Q., Chang, S.S., Huang, G., Fisher, D., Cha, J., Poranen, M.M., Bamford, D.H., and Liu, Y. (2010). The DNA/RNA-dependent RNA polymerase QDE-1 generates aberrant RNA and dsRNA for RNAi in a process requiring replication protein $A$ and $a$ DNA helicase. PLOS Biol. 8(10): e1000496.

Maeda, I., Kohara, Y., Yamamoto, M., and Sugimoto, A (2001). Large-scale analysis of gene functions in Caenorhabditis elegans by high-throughput RNAi. Curr. Biol. 11: 171-176. 
Malhotra, P., Dasaradhi, P. V. N., Kumar, A., Mohmmed, A., Agrawal, N., Bhatnagar, R. K., and Chauhan, V.S. (2002). Double-stranded RNA-mediated gene silencing of cysteine proteases (falcipain-1 and -2) of Plasmodium falciparum. Mol. Microbiol. 45 (5): 1245-1254

Mc Conkey, G. A., Rogers, M. J., and McCutchan, T. F. (1997). Inhibition of Plasmodium falciparum protein synthesis: Targeting the plastid-like organelle with thiostrepton. J. Bio. Chem. 272: 2046-2049.

Metzke, M., Metzke, A.J.M., and Kooter, J.M. (2001). RNA: guiding gene silencing. Science 293: 1080-1083.

Moissiard, G. and Voinnet, O. (2006). RNA silencing of host transcripts by cauliflower mosaic virus requires coordinated action of the four Arabidopsis Dicer-like proteins. P. N.A. S. 103 (51): 19593-19598.

Ngo, H., Tschudi, C., Gull, K., and Ullu, E. (1998). Double stranded RNA induces mRNA degradation in Trypanosoma brucei. P. N.A. S. 95: 14687-14692.

Onodera, Y., Tanaka, M., and Sato, K. (2001). Inhibitory activity of quinolones against DNA gyrase of Mycobacterium tuberculosis. Antimicrob. Agents Chemother. 47: 447-450.

Pedroni, M. J., Luu, T. N.K., and Lau, A. O.T. (2012). Babesia bovis: A bipartite signal directs the glutamyl-tRNA synthetase to the apicoplast. Exp. Parasitol. 131: 261-266.

Suarez, C.E. and McElwain, T.F. (2008). Transient transfection of purified Babesia bovis merozoites. Exp. Parasitol. 118: 498-504.

Suarez, C.E. and McElwain, T.F. (2009). Stable expression of a GFP-BSD fusion protein in Babesia bovis merozoites. Internat. J. Parasitol. 39: 289-297.

Suarez, C. E. and McElwain, T. F. (2010). Transfection systems for Babesia bovis: A review of methods for the transient and stable expression of exogenous genes. Vet. Parasitol. 167: 205-215.
Suarez, C.E., Lacy, P., Laughery, J., Gonzalez, M.G., and McElwain, T. (2007). Optimization of Babesia bovis transfection methods. Parasitologia, Suppl. 1: 67-70.

Tabara, H., Grishok, A., and Mello, C.C. (1998). RNAi in C. elegans: soaking in the genome sequence. Science: 282, 430-431.

Ullu, E. and Tschudi, C. (2000). RNAi-tool in parasitology. New Tech. Life Sci. 6, 43-46.

Vial, H.J. and Gorenflot, A. (2006). Chemotherapy against babesiosis. Vet. Parasitol. 138:147-160.

Waters, A.P., Thomas, A.W., Van Dijk, M.R., and Janse, C.J., (1997). Transfection of malaria parasites. Methods 13: 134147.

Yu, L., Gao, Y-F., Li, X., Qiao, Z-P., and Shen, J-L. (2009). Double-stranded RNA specific to adenosine kinase and hypoxanthine-xanthine-guaninephosphoribosyl transferase retards growth of Toxoplasma gondii. Parasitol. Res. 104: 377-383

Xue, X., Zhang, Q., Huang, Y., Feng, L., and Pan, W. (2008). No miRNA were found in Plasmodium and the ones identified in erythrocytes could not be correlated with infection. Malaria J. 7: 47-52. 